development of the grain. The rate of growth will probably be slow.

Current experimental techniques hold out hope of obtaining the necessary data for carrying out a detailed analysis according to the method outlined.

This research has been supported by the $\mathrm{Na}$ tional Science Foundation and Atomic Energy Commission.

Ruedenberg, K. and Scherr, C. 1953, J. Chem. Phys. 21, I 565 .

Department of Physics and Enrico Fermi Institute for Nuclear Studies, University of Chicago, Chicago, Ill.

Schwartz, Sandra. The inhomogeneous Milne
equation.

The mathematical analysis of the transfer of integrated radiation through a semi-infinite grey atmosphere stratified in plane layers leads to the inhomogeneous Milne equation:

$$
J(\tau)-\int_{0}^{\infty} E_{1}(|t-\tau|) J(t) d t=k(\tau)
$$

where $J(\tau)$ is the source function and $k(\tau)$ is $-\frac{1}{4}$ times the divergence of the flux. In the study of monochromatic radiation transfer, whether or not the atmosphere is grey, a similar equation will appear, containing an additional parameter which corresponds to the frequency of the radiation. This will not affect the form of the solution.

The divergence of the integrated flux is unequal to zero in those parts of the atmosphere which are not in radiative equilibrium: e.g., the regions where convection or energy generation takes place, or where external illumination is received. The divergence of the net monochromatic flux is also a function of the depth in the atmosphere since the atmosphere cannot be expected to be in radiative equilibrium with the radiation of each frequency.

Another problem yielding a non-vanishing flux divergence term arises when one analyzes the flow of radiation in terms of the diffusion of photons. On approaching the stellar surface the flux of photons continually increases because of the breakup of high-frequency photons into photons of lower frequency.

The physical theories of photon diffusion and radiative transfer corresponding to the case of a non-vanishing inhomogeneous term have not yet been developed to the point where an explicit form for that term can be found. However, gen- eral considerations indicate that at least two types of expansions of $k(\tau)$ will be appropriate to these problems: one, in terms of decreasing exponentials (Problem A); the other, in terms of Laguerre polonomials of order $2 m$, multiplied by the function $e^{-\tau / 2} \tau^{m}$ (Problem B).

The procedure that is used to solve for the explicit form of the source function corresponding to problems $\mathrm{A}$ and $\mathrm{B}$ is modeled after the solution of the homogeneous equation and begins with the derivation of the Laplace transform of the source function, i.e., the limb darkening function, for the assumed expansions of $k(\tau)$. The source function itself is then found by inverting the Laplace transform. Observations of the limb darkening together with an assumed expansion for $k(\tau)$ will suffice to find the values of the coefficients in the expansion as well as the source function itself.

It is found that the function $\mathrm{H}(\mu)$ which gives the limb darkening for integrated radiation in the case of strict radiative equilibrium also appears as a factor in the limb-darkening functions corresponding to problems A and B. Due to the generality of the assumed expansions, and the myriad transfer problems they can represent, the presence of this function can be seen to arise simply from the mathematical similarity of the homogeneous and inhomogeneous equations and does not appear to have any physical significance, contrary to a surmise made by some authors.

In principle, it should be possible to apply this analysis to the observed limb darkening in the sun, and using the assumption of local thermodynamic equilibrium, obtain more exact knowledge of the regions of convection.

Yale University Observatory, New Haven, Conn.

\section{Searle, Leonard. A study of three shell stars.}

The spectra of three shell stars have been studied from high dispersion plates obtained at the roo-inch telescope of Mount Wilson Observatory. The stars involved are I Delphini, HD33232 and HDi95407. The U, B and V colors were measured photoelectrically at the 60-inch telescope. From plates at a dispersion of 4.9 $\mathrm{A} / \mathrm{mm}$, which were available for I Delphini, line profiles of $27 \mathrm{Fe}$ II, $\mathrm{Ti}$ II and $\mathrm{Cr}$ II lines were measured. Equivalent widths of all unblended shell-absorption lines were measured and ionic abundances derived.

In all three stars dilution effects are promi- 